\title{
Grouping discontinuities in representative sets: influence on the stability analysis of slope cuts
}

\author{
A. T. Nguyen ${ }^{1,2} \cdot$ V. Merrien-Soukatchoff ${ }^{3} \cdot$ M. Vinches ${ }^{4} \cdot$ M. Gasc-Barbier ${ }^{5}$
}

\begin{abstract}
Based on their orientation, different methods of grouping discontinuities in sets can be used; thus, three grouping methods and their influence on subsequent stability analyses are compared in this study. The first method combines the approaches of Priest, Shanley, Mathab and Yegulalp; the second method consists of grouping discontinuities manually based on an examination of density contour plots; and the third method is based on the spectral method. Unlike the others, the first method allows for the automatic determination of the number of discontinuity sets. The first and third methods were programmed in the Mathematica software environment, while DIPS software was used for the second method. Depending on the grouping process used, each discontinuity or only a portion of all discontinuities is assigned to a set. Therefore, the results in terms of the number of sets; the mean and dispersion of the sets; and the spacing of a given set differ
\end{abstract}

V. Merrien-Soukatchoff

veronique.merrien@lecnam.net

A. T. Nguyen

anh-tuan.nguyen@univ-lorraine.fr

M. Vinches

marc.vinches@mines-ales.fr

M. Gasc-Barbier

muriel.gasc@cerema.fr

1 GeoRessources, École des Mines de Nancy, Université de Lorraine, Lorraine, France

2 Faculty of Mining, Hanoi University of Mining and Geology, Hanoi, Vietnam

3 Geodesy, Geomatics, Geosciences, Planning and Land Law Laboratory $(\mathrm{GeF})$, Cnam, Paris, France

4 Laboratoire LGEI, École des Mines d'Alès, Alès, France

5 Cerema, Toulouse, France with different methods. The consequences of a statistical analysis of orientation on the stability analysis were studied with simulations using RESOBLOK software, which couples the construction of 3D geometric block systems and a quick, iterative, limit-equilibrium stability analysis. Different fracture networks were generated with variable input parameters that were derived from previous statistical analyses of orientation and spacing. The software provides statistical outputs, such as the number and volume of unstable blocks, that can be compared across different cases. An application in a cutting slope near Ax-les-Thermes is presented; the influence of the grouping methods and of the uncertainty of slope orientation on stability indicators is presented. A synthetic indicator of global stability is also proposed.

Keywords Discontinuities - Grouping of discontinuities . Discrete fracture network (DFN) · Modelling · Stability analysis

Résumé Il existe différentes méthodes de regroupement des discontinuités en familles en fonction de leur orientation. Trois méthodes de regroupement sont comparées ainsi que leur influence sur l'analyse de stabilité. La première méthode combine les approches de Priest, Shanley, Mathab et Yegulalp. La seconde consiste à regrouper "manuellement" les discontinuités en se basant sur les contours d'isodensité de fractures et la troisième se base sur la méthode spectrale. La première et la troisième méthode ont été programmées dans l'environnement Mathematica tandis que le logiciel DIPS a été utilisé pour le regroupement "manuel". Selon la méthode de regroupement utilisée, l'ensemble des discontinuités ou seulement une partie d'entre elles sont affectées à une famille. La moyenne et la dispersion des orientations, ainsi que l'espacement entre 
fractures, diffèrent d'une méthode à l'autre. Les conséquences de l'analyse statistique des orientations sur l'analyse de stabilité sont étudiées par des simulations réalisées avec le logiciel RESOBLOK. Ce code associe une génération géométrique stochastique $3 \mathrm{D}$ d'un massif fracturé et une analyse rapide de stabilité, itérative, basée sur l'équilibre limite. Différents réseaux de fractures peuvent être engendrés en fonction des paramètres d'entrée statistiques issus des regroupements différents en familles basés sur l'orientation et de l'espacement calculé par famille. Le logiciel fournit en sortie des résultats stochastiques tels que le nombre et le volume de blocs instables qui peuvent être comparés d'un cas à l'autre. Une application à un talus en déblai à proximité d'Ax-les-Thermes est présentée: les méthodes de regroupement et les variations possibles de l'orientation du talus influencent les indicateurs de stabilité. Un indicateur synthétique de stabilité global est également proposé.

Mots clés Analyse structurale - Discontinuités · Regroupement des discontinuités · Modélisation · Analyse de stabilité

\section{Introduction}

Rock discontinuities include joints, fractures, faults, and other geological structures, which include any surface, flat or curved, that creates a separation in rock masses (Barton 2012). The properties of discontinuities regarding stability include orientation, persistence, roughness, and infilling (Wyllie et al. 2004). The orientation of a discontinuity can be described by its dip angle and dip direction. The grouping of the discontinuities in primary sets is mainly based on the orientation of the discontinuities, which is a consequence of their genesis; however, other parameters regarding geological, geometric or mechanical aspects have also to be considered. Because stability analyses are generally based on the consideration of families of fractures, how structural orientations aggregate influences the design of engineered slopes. This influence is investigated in this study, while considering uncertainties in the data obtained from a mapping procedure might be more significant than the grouping method selected (Gasc-Barbier et al. 2010).

Structural geologists typically measure and analyse orientation data in rock masses. Classically, the orientation of the discontinuities can be represented graphically using the hemispherical projection method of the normal of the discontinuities, which is called a pole. Synthetized measurements are then used to analyse the instability of excavations due to the presence of discontinuities. The combined orientations of discontinuities determine the shape of the individual blocks composing the rock mass. Generally, simple kinematic analysis assumes the full persistence of discontinuities throughout the slope, but the persistence of the discontinuities can possibly be measured and considered using a Discrete Fracture Network (DFN) model.

Several methods have been proposed to gather orientation data and calculate the average/representative orientation of each major discontinuity set. However, none is widely accepted, and some depend on the user's skill and background. After partitioning the fracture data, each set can be characterized by a central tendency quantity, which can be the mean, the median, or the mode of the orientations, and the dispersion around this central tendency. The practitioner can judge whether the median or the mode is the most representative measure compared to the mean for a particular location; however, how to find discontinuity sets of orientation data and "get an effective statistical analysis of sets of orientation data remains a great challenge" in rock discontinuity engineering ( $\mathrm{Wu}$ and $\mathrm{Zhang}$ 2013).

The first method of grouping, which is based on the isodensity curves of poles in a stereographic net, was proposed by Schmidt and Kamb (Priest 1993). Mahtab, Shanley and Yegulalp developed counting techniques to identify clusters of orientation data that strongly depended on the density of data points; they also sought to minimize an objective function to identify clusters of orientation data (Shanley and Mahtab 1976; Mahtab and Yegulalp 1982), as developed in "Methods for defining primary discontinuity sets" section. Harrison initially proposed solving the problem of separating discontinuities into sets using the fuzzy $k$-mean algorithm (Hammah and Curran 1998), which attempts to locate regions of high density in orientation data. The centroids (i.e., mean) of each cluster, which is a cluster's most representative vector, is the geometric mean of all of the vectors belonging to that cluster. Pecher (1989) developed and supervised an expert method for grouping directional data distributions. First, a contour density plot is calculated; then, an observer picks initial values for the mean orientation of one to a maximum number of clusters. The initial guesses are then refined using the fitting method for the cluster by the principal eigenvectors of the orientation matrices (Pecher 1989). Hammah and Curran described a related approach that is based on fuzzy sets and on a similarity measure, which is used by the data analyst to obtain objective and accurate answers in the task of delineating clusters (Hammah and Curran 1998, 1999). However, Klose et al. (2005) proposed a clustering approach for the partitioning of directional data, which was based on vector quantification. Directional 
data are grouped into separate isotropic clusters and concurrently, the average dip direction and dip angle are calculated for each group. More recently, Jimenez-Rodriguez and Sitar, and Rafiee and Vinches used the spectral clustering approach with a measure of similarity between normal unit vectors in the spherical space (Jimenez-Rodriguez and Sitar 2006a; Rafiee and Vinches 2008). In this method, each discontinuity is first represented by its unit normal vector on the equal-area lower-hemisphere projection of a unit reference sphere. Then, a sine-based similarity measure between each pair of these vectors is calculated using the acute angle in Cartesian coordinates. Lastly, a transformation of the unit normal vectors from the Cartesian space into the $\mathrm{k}$-dimension space is executed based on the affinity conversion and $k$-mean algorithm. Spectral clustering algorithms that group points using eigenvectors of matrices that are derived from orientation data are based on the work of Andrew et al. (Andrew et al. 2002; Meila and Shi 2001). However, one of the limitations of the methods listed above is that the number of desired clusters must be defined in advance, while this decision is subjective and depends on the expert.

Because there is no widely accepted method for grouping discontinuities in sets, we present and develop three methods of grouping fractures that affect rock masses into discontinuity sets and observe the subsequent consequences on slope stability analyses. Two grouping methods were programmed in Mathematica version 9.0 software and were linked to a standalone application by Wolfram Research (1988); only the standard Mathematica functions with a registered trademark of The Wolfram Research, Inc. were needed. These Mathematica scripts were developed for clustering orientation data, visualizing the results, and presenting statistical summaries. We also used DIPS (Data Interpretation Package using Stereographic projection) version 5.1 software (2003) of Rocscience Inc. for the plotting, analysis, and presentation of structural data. Each of the three methods computes mean orientation and dispersion around the mean for each of the proposed sets. These statistics allow stochastic simulations of geometries of rock discontinuities. The influence of the grouping algorithm on the stability analysis is examined using RESOBLOK software (Heliot 1988; Merrien-Soukatchoff et al. 2012), which allows a stochastic discrete fracture network (DFN) model to be built based on a statistic knowledge of discontinuities orientation, persistence, and spacing.

The differences in the grouping methods and the consequences on stability analyses are shown using the case of a granitoid migmatites slope cutting of a road near Ax-lesThermes in southern France. The site was chosen due to its easy access to discontinuity orientations and slope cut geometries, and a detailed geological description of the case provided by Gasc-Barbier et al. (2008), which is summarized in "Application to a slope-cutting" section. A first grouping into families was also proposed in a previous study.

\section{Methods for defining primary discontinuity sets}

\section{Discontinuity orientation}

The data of discontinuities and their parameters are typically acquired via core logging, scan lining, window mapping of rock exposures, and other methods (Priest 1993). The orientation of a discontinuity is defined by its dip angle $\left(\beta_{d}\right)$ and its dip direction $\left(\alpha_{d}\right)$ [Fig. 1 Parameters defining the discontinuity orientation of a plane (Wyllie et al. 2004)].

The trend $\left(\alpha_{n}\right)$ and plunge $\left(\beta_{n}\right)$ of the normal (i.e., downward directed end) to a given plane of discontinuity are related to the dip angle and direction of the plane by: $\alpha_{n}=\alpha_{d} \pm 180^{\circ} ;\left(0^{\circ} \leq \alpha_{n} \leq 360^{\circ}\right)$ and $\beta_{n}=90^{\circ}-\beta_{d}$.

The normal of a plane can also be represented by a unit vector $X^{T}\left(u_{x}, u_{y}, u_{z}\right)$ in a Cartesian coordinate system, where $x$ corresponds to the North direction, $y$ to the West direction and $z$ to the vertical. Adopting the tri-dimensional system proposed by Priest, these coordinates are given by Eq. (1):

$$
\left\{\begin{array}{c}
u_{x}=\cos \left(\alpha_{n}\right) \cos \left(\beta_{n}\right) \\
u_{y}=\sin \left(\alpha_{n}\right) \cos \left(\beta_{n}\right) \\
u_{z}=\sin \left(\beta_{n}\right)
\end{array}\right.
$$

Hemispherical projections based on equal angles or equal areas (Wülff or Schmidt) can also be used to plot discontinuity data. In this study, we use a lower hemisphere Schmidt net to represent the orientation data.

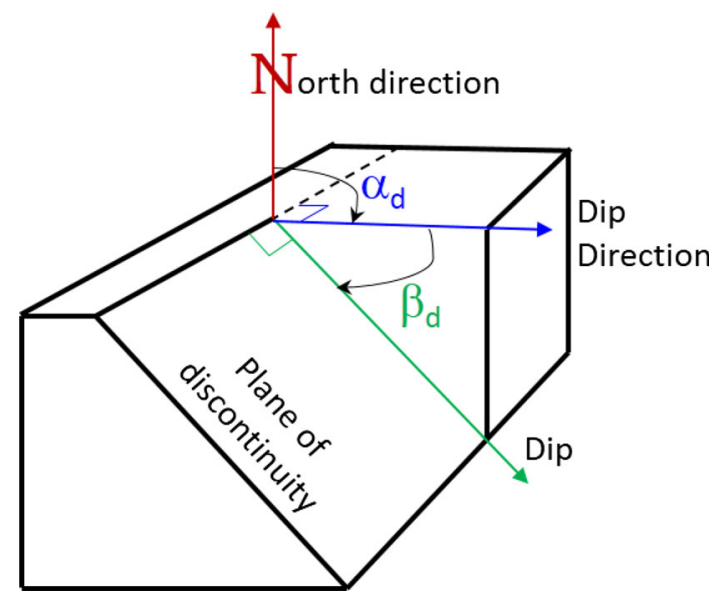

Fig. 1 Parameters defining the discontinuity orientation of a plane (Wyllie et al. 2004) 


\section{Grouping discontinuities into primary discontinuity sets}

Let us consider an orientation dataset with $N$ measurements of rock discontinuity orientation, where $N=\left\{\left(\alpha_{d 1}, \beta_{d 1}\right)\right.$, $\left.\left(\alpha_{d 2}, \beta_{d 2}\right), \ldots,\left(\alpha_{d N}, \beta_{d N}\right)\right\}$ is a list of dip directions and angles. We aim to group $N$ orientations of the discontinuities into $K$ discontinuity sets. Three possible grouping algorithms are presented below and are then applied to a slope cut.

\section{PSMY method}

By combining the approaches of Priest, Shanley, Mathab and Yegulalp (Priest 1993; Shanley and Mahtab 1976; Mahtab and Yegulalp 1982), we developed a Mathematica script called PSMY, which was named based on the authors whose methods were combined to create it. The script allows visualizing, analysing, and clustering the data, and displays statistical summaries from the orientation dataset. The PSMY script considers that the frequency of poles is greater in zones of concentrations of discontinuity poles compared to it would be if the process was completely random, where a random process is expressed by a Poisson Law.

The proposed script involves the following steps:

- In a cone with an angle $\delta$ between its axis and surface, it is possible to compute the frequency of discontinuities associated with random events following a Poisson Law. The calculation of this critical frequency $t_{\text {crit }}$ for a given $\delta$ angle allows the identification of zones of pole concentration, where the effective frequency is greater than this critical frequency. This calculation can be performed with an angle $\delta$ centred on each normal to a discontinuity.

- The calculation of angle $\delta_{i j}$ between each normal to a discontinuity ( $i$ ) and all of the normals to another discontinuity $(j)$ allows for the determination of the number of "neighbours" of each discontinuity inside a cone of the given angle $\delta$. This step permits the identification of the discontinuity with the largest number of neighbours for a given angle $\delta$. The number of neighbours thus depends on the chosen acute angle $\delta$.

- After "eliminating" the discontinuity with the largest number of neighbours, the previous stage is repeated on the remaining discontinuities in an iterative way if the number of neighbours remains superior to $t_{\text {crit }}$; thus, $p$ "dense points" are reached, where each $p$ dense points and its neighbours form "a class".

- A pole corresponding to the centre (i.e., mean of the orientation) of each of the $p$ classes is then computed.

- The next step consists of grouping the same set of classes, such as the acute angles between the centres that are lower than $\delta$. This step will reduce the number of classes from $p$ to $k$ (i.e., $k$ sets) and the mean of these $k$ sets is computed again (i.e., new dense points).

- Finally, all the non-dense points, which are inside the $\delta$ angle of a dense point, are allocated to the group of the dense point.

At the end of this stage, $k$ sets with their mean orientation and the standard deviation angle of the set are obtained. Certain discontinuities can be "left out" (i.e., not assigned to any of the defined sets); the proportion of discontinuities not belonging to a family depends on the $\delta$ angle.

Because the partition and the number of sets depend on the chosen angle $\delta$, an "objective function" $(F(\delta))$ based on the Huygens decomposition, as suggested by Shanley, is considered to select the best partition of the discontinuities (Shanley and Mahtab 1976):

$F(\delta)=\sum_{j=1}^{k} \sum_{i=1}^{N_{j}} d^{2}\left(X_{j}^{i}, \bar{X}_{j}\right)+\sum_{i=1}^{k-1} \sum_{j=1+1}^{k} d^{2}\left(\bar{X}_{i}, \bar{X}_{j}\right)$,

where $k$ is the number of clusters in partition; $N_{j}$ is the number of points in cluster $j ; X_{j}^{i}$ is the $i$ th element of cluster $j ; d\left(X_{i}, X_{j}\right)$ is the distance between $X_{i}$ and $X_{j}$; and $\bar{X}_{i}$ is the centre of gravity of cluster $i$.

For calculating the distances between the individuals of the same class, we use the Euclidean distance, which is the distance between two vectors that are normal units of discontinuity $X_{i}\left(u_{x i}, u_{y i}, u_{z i}\right)$ and $X_{j}\left(u_{x j}, u_{y j}, u_{z j}\right)$, and is described by:

$d^{2}\left(X_{i}, X_{j}\right)=\left(u_{x i}-u_{x j}\right)^{2}+\left(u_{y i}-u_{y j}\right)^{2}+\left(u_{z i}-u_{z j}\right)^{2}$.

The minimum of the objective function relative to $\delta$ can be obtained (Mardia 1975; Picard 1999; Mirkin 1996) by testing different $\delta$ values. This method was programmed in the Mathematica software environment, and the graph of the variation of the objective function versus $\delta$ was also programmed in this environment.

\section{Spectral method}

Andrew et al. and Jimenez-Rodriguez and Sitar initially proposed a spectral grouping method (Andrew et al. 2002; Jimenez-Rodriguez and Sitar 2006a) that must fix the number $K$ of clusters to be used. If $K$ is chosen as the number of families identified by the PSMY method, then the number of families to be considered may be automatically specified. In the subsequent example ("Structural study and statistical treatments" section), two $K$ values will be proposed to make comparisons between the PSMY and spectral methods and between the two options of the spectral method. 
The spectral grouping algorithm transforms the unit normal vectors from the Cartesian space into the $K$-dimension space, where $R^{K}$ and $K$ refer to the number of clusters, using the eigenvectors of the matrices of similarity between the data points and the $K$-means algorithm (Jimenez-Rodriguez 2008; Andrew et al. 2002). The $K$ means algorithm calculates the distance between each pair of points and works iteratively in two steps. First, if the number of data is bigger than the number of clusters $K$, the initial mean locations can be the first $K$ points, or can be taken randomly as $K$ distinct points. Then the mean locations are calculated again for the $K$ groups. In a second step, for each of the data, the distances to the mean of each of the clusters are calculated and the minimum distance is recorded. This data is then assigned to the cluster whose mean value corresponds to this minimum distance. This process is repeated until no data moves to another cluster.

The spectral method uses an adjusted parameter $(\sigma)$ that will not be detailed in this study but influences the studied case presented in the "Structural study and statistical treatments" section. Based on Jimenez et al., the optimal value of $\sigma$ depends on the selected distance measure and on the dataset used; Jimenez et al. also suggest that it should be chosen in the order of $0.1-0.15$ (Jimenez-Rodriguez and Sitar 2006a). Several values have been tested in the studied case below.

This method was also programmed and presented using Mathematica software.

Using DIPS ${ }^{T M}$ software

DIPS is a commercial software developed by Rocscience (2003) that calculates the hemi-spherical projections and stereograms of poles of discontinuity densities (Rockscience Inc. 2003), which characterize the percentage of points of the diagram contained in 1/100th of the total surface for a given orientation data (Diederichs 1990). It is possible to group the discontinuities in the primary families around zones with high fracture densities. The boundaries of the groups are chosen by the user of the software.

\section{Statistics of the different primary discontinuity sets}

Priest, and Hammah and Curran detail how to compute the mean orientation and the dispersion around this mean of the discontinuities belonging to the same discontinuity set (Priest 1993; Hammah and Curran 1998). The distribution of the poles based on a given direction is typically adjusted using the Fisher distribution (Kabbaj and Baroudi 1995), which leads to the determination of the average orientation of the family and its dispersion using the Fisher coefficient $\left(K_{f}\right)$ (Priest 1993).
Discontinuity spacing, which is perpendicular to the mean orientation, can then be evaluated in the field if each discontinuity can be a priori allotted to a fracture sets or can be calculated within each discontinuity set based on the location of each discontinuity. The spacing is generally fitted to a negative exponential, log-normal, or gamma law depending on their mean and standard deviation. If the discontinuity spacing follows a negative exponential law, it is characterized with a single parameter $\lambda$, which is the inverse of the mean spacing.

The knowledge of the set of discontinuities and their associated statistics in orientation and spacing can be used as a base to represent a fractured rock mass in a DFN model and then to run stability computations. The influence of grouping on stability analyses is shown in the next section.

\section{Application to a slope-cutting}

\section{Structural study and statistical treatments}

Discontinuity surveys were performed before cutting of a slope for the Ax-les-Thermes diversion road detailed in many references (Merrien-Soukatchoff et al. 2012; Godefroy et al. 2009; Gasc-Barbier et al. 2008). Ax-les-Thermes city (Fig. 2), in the Pyrenees mountain, is approximately $130 \mathrm{~km}$ south of Toulouse and $30 \mathrm{~km}$ from the border between France and Andorra in southern France.

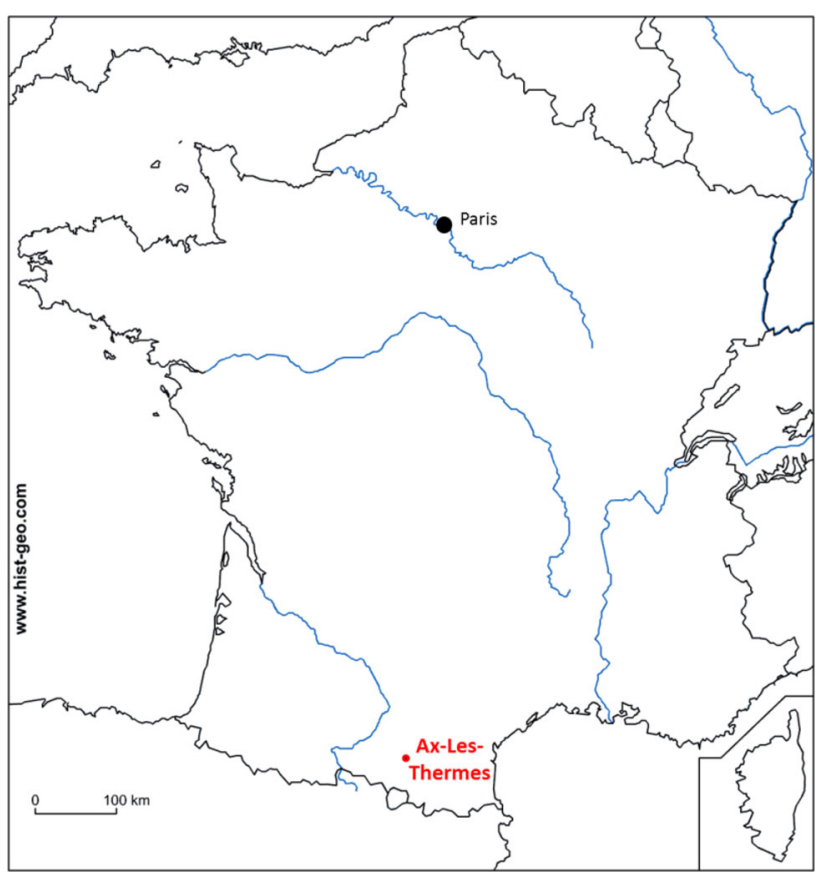

Fig. 2 Location of Ax-les-Thermes on a map of France (modified from http://www.hist-geo.com/Fond-de-carte/France/France-Fleuves1.php) 
The Pyrenees mountain separates France from Spain in a relief whose width varies between 50 and $150 \mathrm{~km}$. These mountains were created by three successive orogenies (i.e., Hercynian stage -330/-280 Ma, Alpine stage -115/-40 with its paroxysm during the Lutecian stage $-50 /-40 \mathrm{Ma}$, and finally the Mediterranean stage $-25 /-6 \mathrm{Ma}$ ), which produced highly fractured rocks and high-temperature lowpressure metamorphism that affected the sediments.

The French Pyrenees are typically organized into three areas:

- the axial primary zone, which consists of Hercynian rocks (i.e., gneiss, granitic massif and micaschists), that is bounded to the north by the North Pyrenean fault;

- the North Pyrenean area, which consists of rocks that are primarily from the secondary era with certain Hercynian massifs; and

- the sub-Pyrenean area, which is as a Foreland and primarily consists of Cretaceous and Tertiary rocks.

Ax-les-Thermes is located in the axial primary zone (Fig. 3); thus, the bypass encountered successively granite, granitoid migmatite, and gneiss from the Upper Carboniferous era, which was highly fractured and recovered in the valley by Quaternary fluvio-glacial deposits of variable thickness. The geological description of the site is detailed by Gasc-Barbier et al. (2008) in this journal.

The studied slope cuts a granitoid migmatite. It is between 22 and 37 metres high with a dip angle of $70^{\circ}-76^{\circ}$ and a variable azimuth due to a change of direction in the bypass road from South-North to East-West. A total of 856 discontinuities were collected on 11 scan lines that were performed on different orientations and cuttings (Fig. 4).

Discontinuity spacing was evaluated in the field by assigning a priori a fracture to a set; however, the location of each discontinuity was also measured.

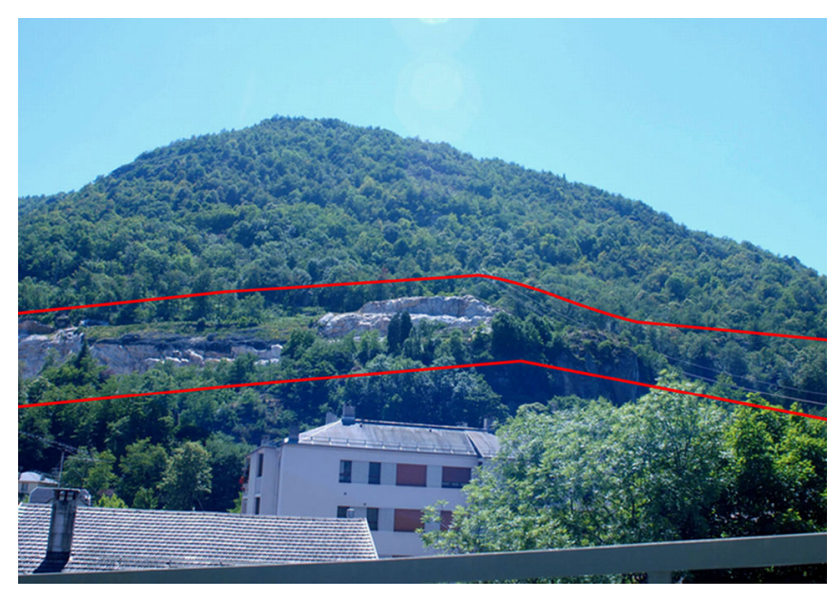

Fig. 3 Overview of the bypass, viewed from Ax-les-Thermes city

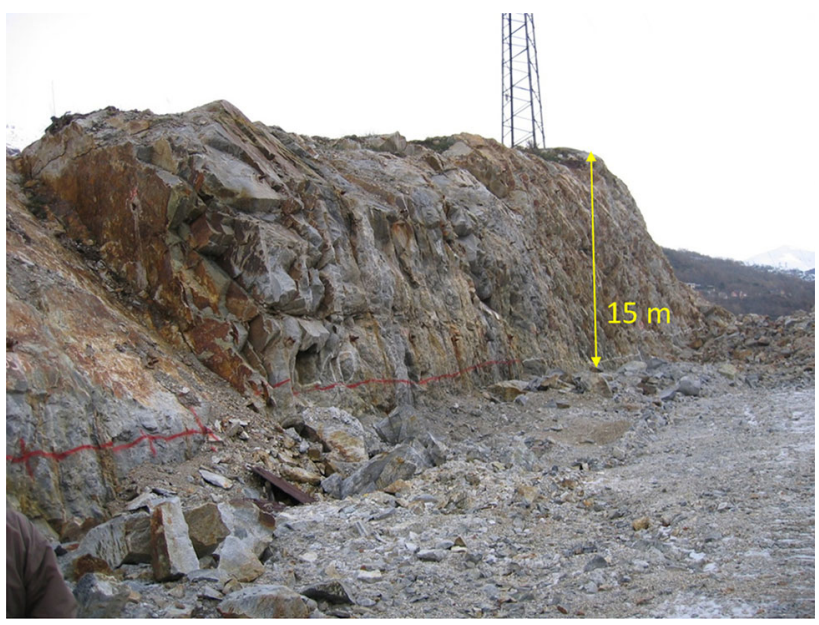

Fig. 4 Slope cut during excavation, showing a horizontal scan-line measurement

The type, orientation, persistence, roughness and location of each discontinuity intersecting each scan line were reported with an estimated perpendicular distance while considering an a priori family of each fracture when reporting the data and estimating the perpendicular distance of each fracture to the nearest fracture of the same family.

Examining the poles reported on an equal-area lowerhemisphere projection. (Fig. 5a); any a priori partition in families is thus evident, and the poles seem to be dispersed over the entire diagram. Most of the fractures dip steeply but their orientation seems almost equally distributed.

The three grouping methods presented above were applied to divide the discontinuities into sets. Figure 5 displays the different sets resulting from the run of the different classification methods on an equal-area lowerhemisphere projection. Most of the methods used in this study did not classify all of the fractures; the boundaries of classes are delimited, but certain fractures do not belong to any group. The percentage of fractures classified was considered to be the "level" of the method. The methods were ranked based on increasing "levels" of consideration of the existing discontinuities (i.e., from 50 to $100 \%$ ).

1. Different manual groupings were proposed using DIPS software (level I Ni in Table 1). This procedure yields a variable grouping rate from 50 to $85 \%$ in three or four sets based on the assumed limits of the families. A manual regrouping was previously proposed by GascBarbier et al. (2008) with $50 \%$ of the data classified (Dips Ni_case1); also, based on additional measurements on outcrops, Gasc-Barbier et al. (2008) also proposed a fourth family, which was not intersected by the scan-lines (Dips Ni_case4 in Table 1). We considered two other possible regroupings in three or four 
Fig. 5 Stereographic projection in a lower-hemisphere Schmidt net: a plot of discontinuity data of the 11 scan lines (one colour/ scan line); b manual groupings into three and four primary sets using the DIPS method: Dips(Ni)_case1 with approximately $50 \%$ of the points belonging to no family (Gasc-Barbier et al. 2008); Dips(Ni)_case2 with approximately $41 \%$ of the points belonging to no family; and $15 \%$ of the points belonging to no family in Dips(Ni)_case3 with four primary sets; $\mathbf{c}$ three primary families using the PSMY method with $47 \%$ of the points belonging to no family; $\mathbf{d}$ three primary families using the spectral method; e four primary families (experimental) using the spectral method; f comparison of the pole sets using the spectral method, where $\sigma$ equals 0.21 for these two cases; and $\mathbf{g}$ comparison of the pole sets of the various grouping methods

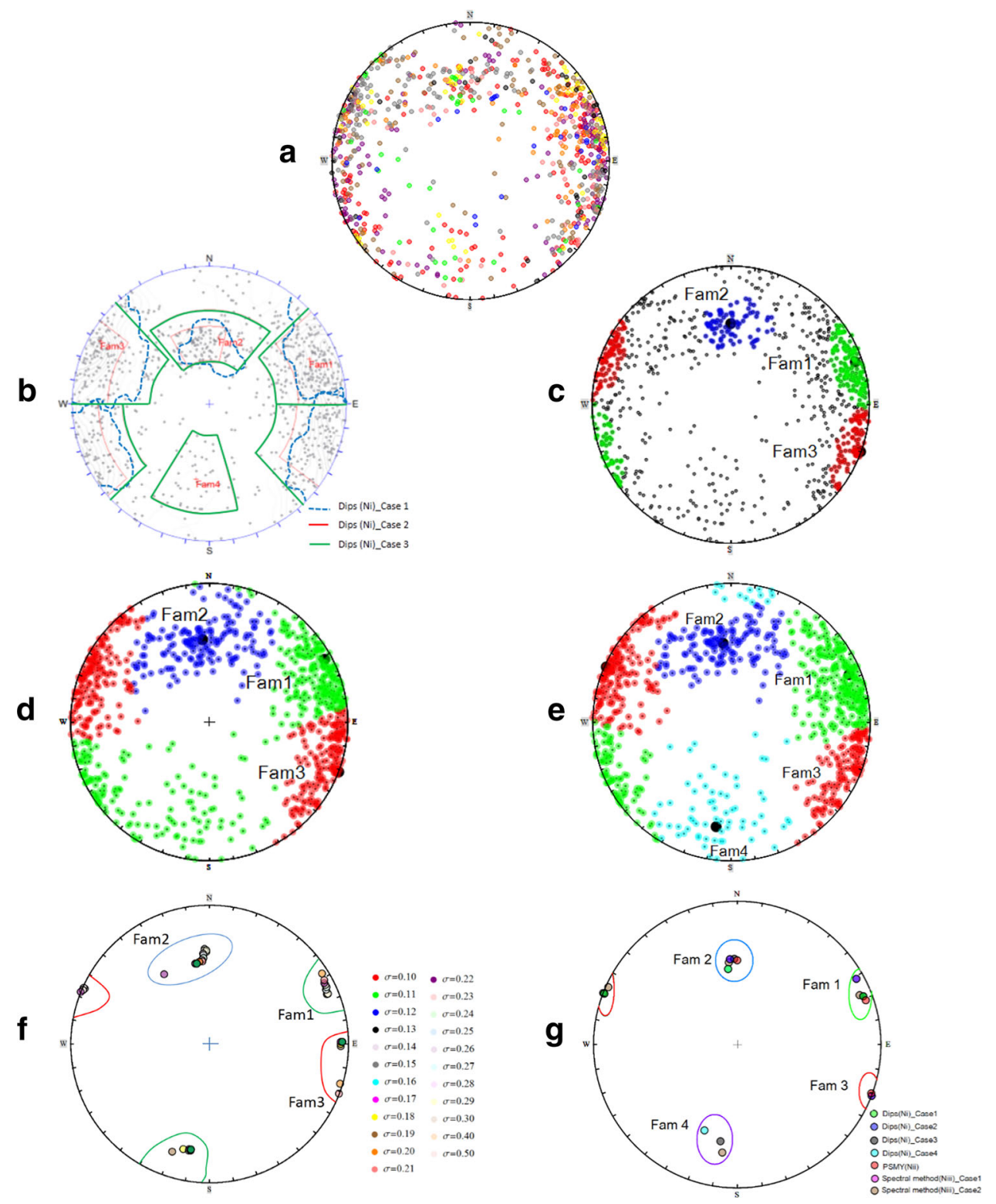

families (i.e., cases 2 and 3), and the case corresponding to three joint sets is displayed on Fig. 5b; the groups Dips Ni_case2 and Dips Ni_case3 grouping classifying, respectively, 59 and $85 \%$ of the discontinuities. Using this manual aggregation mode, the percentage of classified fractures was quite variable based on the limits considered. The spacing of case 4 with the introduction of a fourth family, which was not intersected by the scan-lines, could not be compared to the other cases. The spacing used was greater than those observed on the scan lines, but the parameters were those previously used by Gasc-Barbier et al. (2008).

2. The PSMY method (level II Nii, Fig. 5c) classified $53 \%$ of the discontinuities. Running the PSMY method and computing the objective function (Fig. 6) based on "PSMY method" section lead to a minimum of the objective function $F(\delta)$ for an acute angle of $\delta=19^{\circ}$ and $t_{\text {crit }}=58$, corresponding to a partition of the discontinuities into three main sets.

3. The spectral method (level Niii) regrouped all of the discontinuities (i.e., $100 \%$ were classified). Case 1 corresponds to the application of the method with three main sets using the number of families determined by PSMY, as shown in Fig. 5d, but an alternative case with four main sets (i.e., case 2) was also investigated (Fig. 5e). In addition, the grouping based on the spectral method depends on a factor $\sigma$ whose sensitivity was investigated by varying $\sigma$ from 0.09 to 0.5 (see Fig. 5f). As mentioned above, the scaling 
Table 1 Detail of discontinuity sets with different data treatment levels for a cutting slope near Ax-les-Thermes

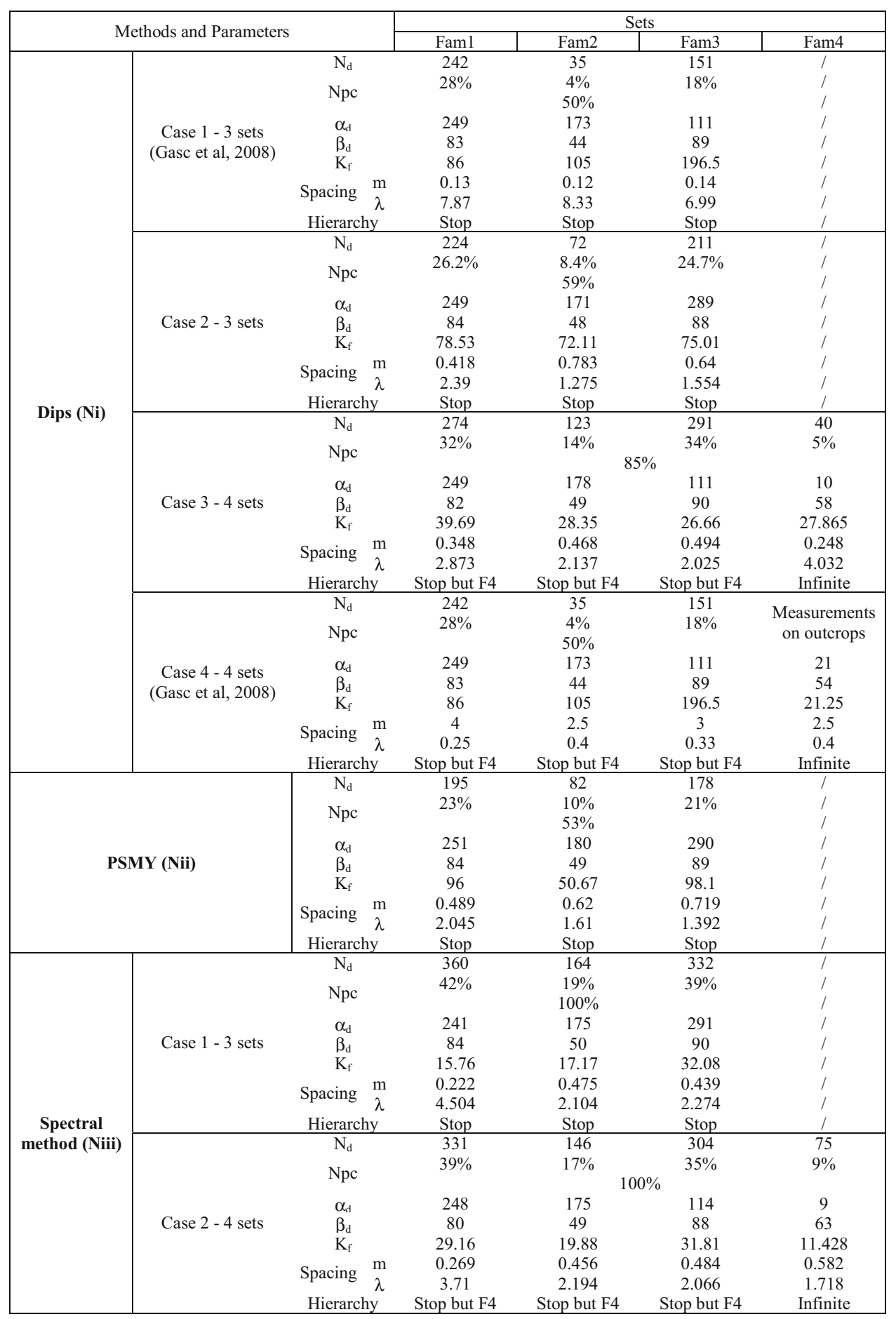

parameter $\sigma$ was a user-specified parameter. As proposed by Meila and Shi (2001), and Andrew et al. (2002), it is possible to choose a value of $\sigma$ that maximises the intra cluster similarity (i.e., low intra- cluster distances). For the proposed case, the $\sigma$ value of 0.21 presented in Fig. 5e is appropriate, but this choice remains debatable between experts (Meila and Shi 2001; Andrew et al. 2002). 


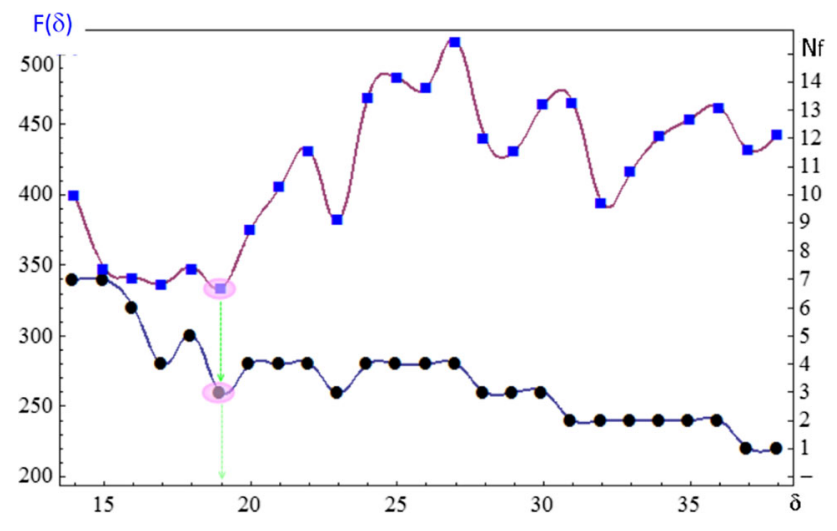

Fig. 6 Number of primary sets $\mathrm{Nf}$ (black) and objective function $F(\delta)$ in blue versus the cone angle $\delta$

No grouping method completely considers the structure of the orientation data (i.e., the shape of clusters in the dataset, the number of observations in the dataset):

- The DIPS grouping is user-friendly and can consider qualitative and geological observations but is userdependent and yields variable levels of data classification. Different data analysts could produce different results based on their background, experience, and personal biases and inconsistencies. The differences are probably more pronounced in cases where the boundaries between primary discontinuities are unclear;

- The PSMY method yields a unique identification of families (Fig. 6) but does not classify all discontinuities, which introduces a subsequent bias on the DFN model; and

- The spectral method depends not only on the number of family sets set by the user, which can be overcome using the number determined by PSMY, but also on the value of the parameter $\sigma$, the determination of which remains under debate.

The poles of sets, depending the various methods of grouping, are displayed in Fig. 5g. They do not appear to be distant from each other. The influence of the various methods of grouping on dispersion, as described by the Fisher coefficient $K_{f}$, is greater (see Table 1), particularly due to the percentage of classified fractures.

The percentage of classified fractures will influence geometric models; when certain discontinuities are not classified into any families, the stochastic geometric simulations resulting from these statistics are biased compared to the original data. A high percentage of classified fractures tend to imply a more accurate geometric model of the corresponding zone.

The Fisher coefficient will also influence geometrical simulations. A low Fisher coefficient tends to imply more scattered stochastic orientations, which will likely lead to more but smaller blocks in the rock mass.
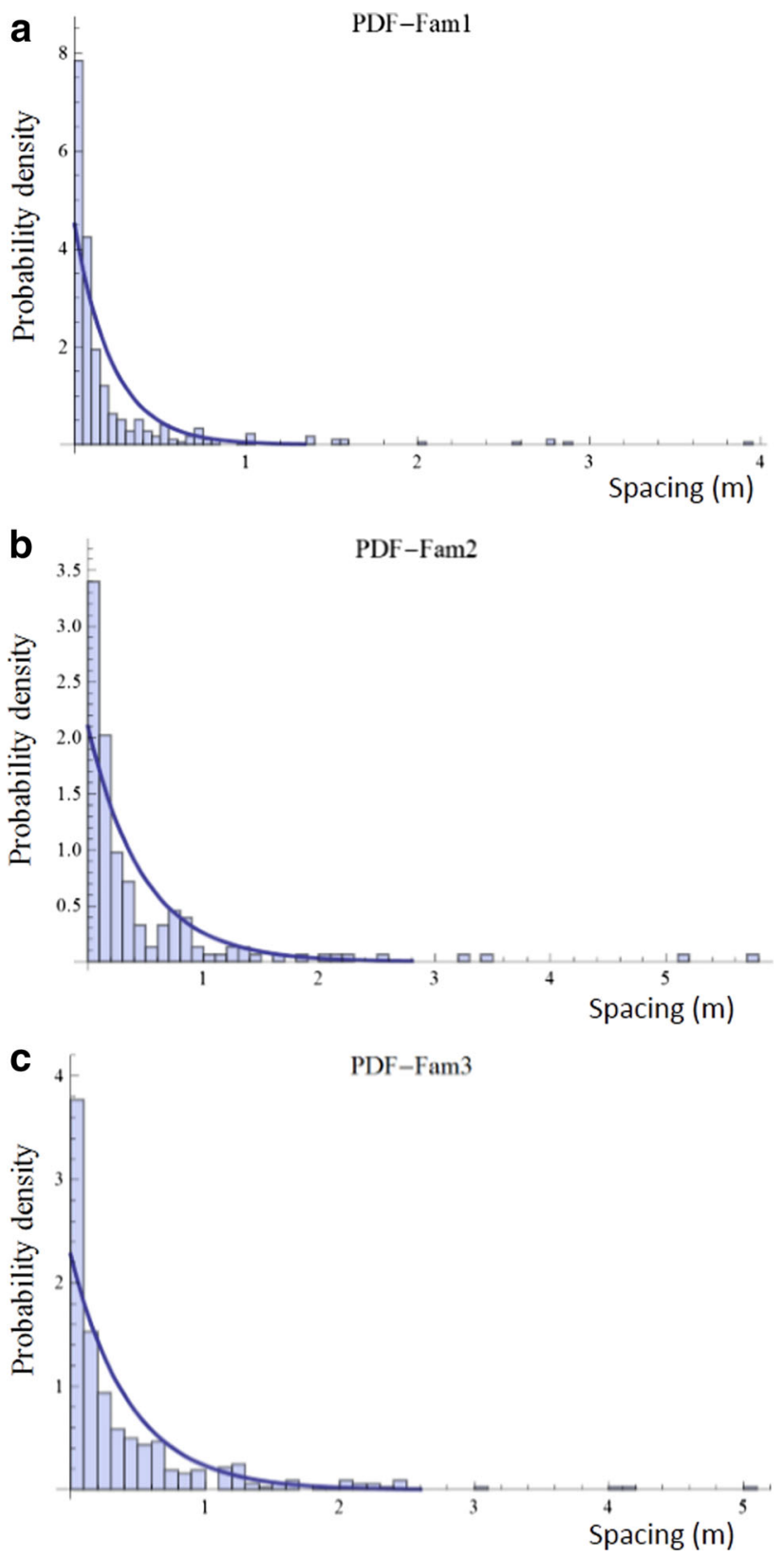

Fig. 7 From top to bottom: histogram and statistical adjustment of the probability density curves of spacing for the three sets at Niii_cas1, family Fam1 (a), Fam2 (b) and Fam3 (c), respectively

When the discontinuities are divided into families, the distance between one discontinuity and another belonging to the same family can be calculated for each scan line. The measured distance along the scan line must be corrected to obtain the normal spacing. The correction factor is the cosine of the angle between the scan line and the mean orientation of the discontinuity set. In Table 1, the (normal) spacing in each family was computed using the location of the discontinuities in the scan lines, except for Dips Ni_case1 and Dips Ni_case4, where the spacing was evaluated in the field. The spacing was considered to follow an 
exponential law (see "Statistics of the different primary discontinuity sets" section) with the parameter $\lambda$ set equal to the inverse of the mean distance (i.e., $=1 / \mathrm{m}$ ). Figure 7 shows the experimental spacing distributions and fitting line, assuming an exponential distribution, for the case Niii_cas1. The $\lambda$ parameter for each family of each case is presented in Table 1 .

Figure 5 and Table 1 show the details of the discontinuity sets, where $N_{d}$ is the number of discontinuities in each family; $N_{\mathrm{pc}}$ is the percentage of data classification; and $K_{f}$ is the Fisher coefficient that characterizes the dispersion. A larger Fisher coefficient tends to imply a lower dispersion.

Figure $5 \mathrm{~g}$ shows that the addition of a fourth family does not significantly change the pole of the other three families. Few fractures belong to this fourth family (see Table 1), which always has a low Fisher coefficient, which thus implies a large dispersion.

The Fisher coefficients produced by the spectral method are significantly lower than those of any of the other regrouping methods; this is caused by allotting all of the discontinuities, including data far from the mean, to a family. For the PSMY or DIPS regrouping methods, the Fisher coefficients are larger than those obtained for the spectral grouping method, and the elimination of part of the discontinuities will inevitably misrepresent the fracture distribution in the following stochastic modelling.

\section{Modelling of fractured slope stability}

\section{RESOBLOK software}

The consequences of the statistical analysis on the stability analysis were studied running RESOBLOK simulations. RESOBLOK is a DFN code (Heliot 1988; Baroudi et al. 1992; Merrien-Soukatchoff et al. 2012) that couples the construction of a geometric block system and an iterative stability analysis based on limit-equilibrium stability analysis and the vector analysis method that was initially proposed by Warburton (Warburton 1981).

Using statistical knowledge of fracture orientations and spacings, block geometries can be computed, and a stability analysis can be performed. For a given geometric scenario, corresponding to a given statistic of fracture orientations and spacings, several different geometric simulations are performed.

For each geometric simulation, three stability (or unstability) indicators are computed: (1) the number of unstable blocks; (2) the mean volume of an unstable block, which is computed using the volume and weight of each unstable block; and (3) the total weight and volume of the unstable blocks.
For a given scenario, and a wide range of simulations, it is possible to display:

- The minimum, average and maximum number of unstable blocks of a given scenario;

- The minimum, average and maximum mean volume of an unstable block of a given scenario; and

- The minimum, average and maximum total volume of the blocks of a given scenario.

This leads to nine global stability indicators for a given scenario. It has been shown (Baroudi et al. 1992) that in most cases, running 50 simulations allows the determination of stable values of the mean and the standard deviation, a histogram of the variable number of unstable blocks, the mean volume of unstable blocks, and the total unstable volume. The evolution of the stability indicators with the number of simulations performed can be determined to confirm the convergence of the stochastic results.

A less stable slope is typically indicated when the minimum, average, and maximum number of unstable blocks and the minimum, average, and maximum total volume of blocks in a given scenario are large. It is more difficult to understand the indicators relative to the mean volume of an unstable block. A priori, it is better to have a small mean volume of an unstable block; however, this can be linked to a large total volume of blocks with a larger number of blocks. The use of the indicators relative to the mean volume of an

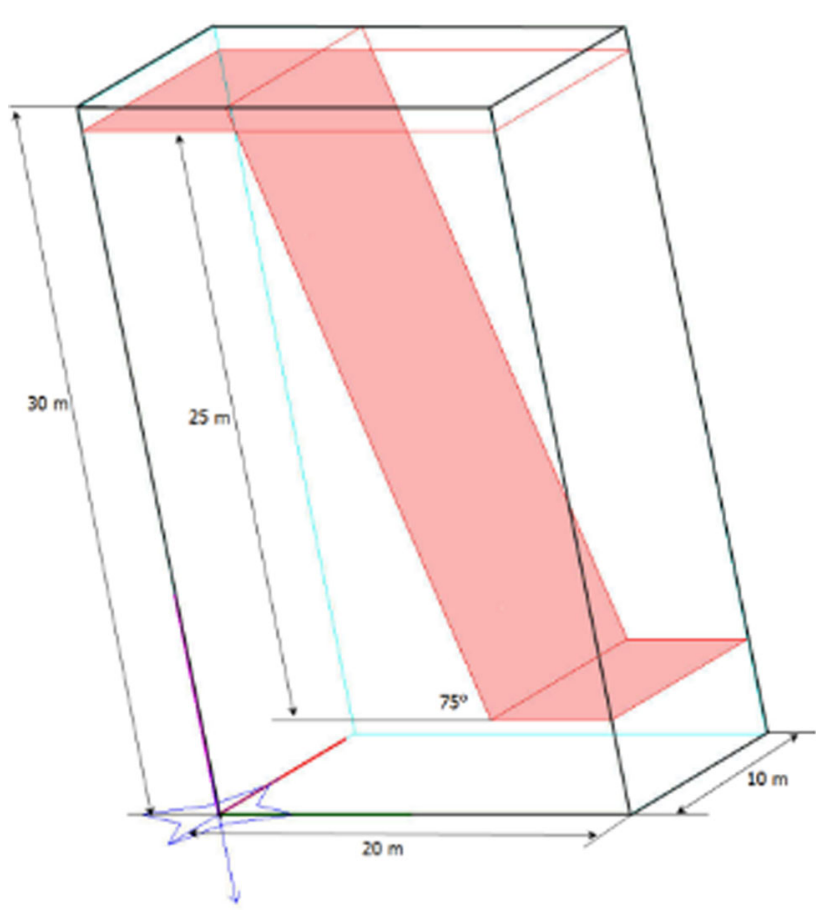

Fig. 8 Ax-les-Thermes cut and model dimensions. Shaded areas are the surfaces of slope cuts in the RESOBLOK model 
Fig. 9 Cross section of the fracturing modelling of the $5^{\circ} \mathrm{N} 75^{\circ}$ bench run with $\mathrm{Ni}$ _cas 4 (four discontinuities sets) statistical parameters, assuming no hierarchical rule (left) and assuming a hierarchical rule $($ right). Dimensions are given in the previous figure
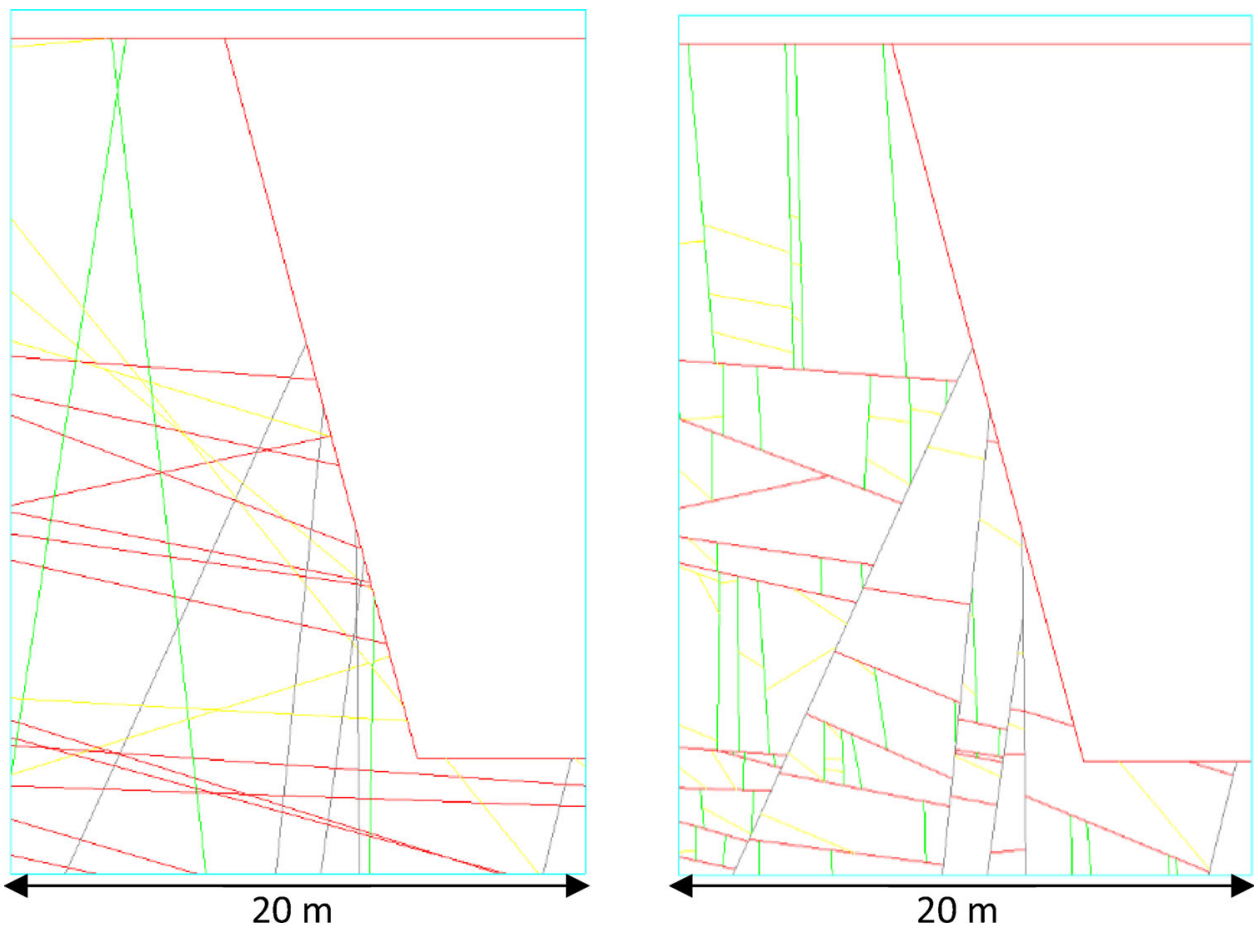

unstable block could depend on the practice used at a given site; many small blocks can be considered to be acceptable.

\section{Geometry of the slope and hierarchical rules}

The studied cut was considered to be $25 \mathrm{~m}$ high with a slope of $75^{\circ}, 10 \mathrm{~m}$ wide, and have an azimuth of $5^{\circ}$. To consider possible variations of the orientation and geometry of the cut, marginal variations of the orientation were set equal to $5^{\circ} \pm 2^{\circ}$. The cases are labelled $(3,75),(5,75)$ and $(7,75)$ (Fig. 8). These different orientations of the slope were combined with the different statistical regroupings of fractures proposed in Table 1.

In addition, RESOBLOK software can consider that a family of fractures cuts the entire zone of interest, or that the family was generated based on a hierarchical process, leading certain families of fractures to end on other families (Merrien-Soukatchoff et al. 2012; Warburton 1981). In RESOBLOK software, fractures must completely cut a previous block; they cannot end in the middle of a block. Because the hierarchical rules of fracturing were not fully available from the site, two different hierarchical rules were considered: either all fractures are stopped by the others, which is reported as "Stop" in Table 1, or all families except $F 4$ are stopped by the others, which is reported as "Stop but $F 4$ " in Table 1 . This alternative was used in this study because it was originally proposed by Gasc-Barbier et al. (2008).
To highlight the influence of the hierarchical rules, Fig. 9 displays the difference on a cross section for a given simulation, while assuming an extension of all fractures throughout the zone of interest (Fig. 9, left) or a hierarchical extension limited to a block (Fig. 9, right). This 2D figure displays the difference more clearly than a 3D picture due to the clarity it provides.

\section{Stability analysis of the cut}

The stability analysis of the cut at the Ax-les-Thermes diversion was performed using the discontinuity sets shown in Fig. 5 and Table 1. The stability analysis only required the input data relative to the rock density $(\gamma=2500 \mathrm{~kg} /$ $\left.\mathrm{m}^{3}\right)$; the cohesion $(C)$ and friction angle $(\varphi)$ of the discontinuities in this study were initially fixed at $0 \mathrm{kPa}$ and $26^{\circ}$, respectively (Merrien-Soukatchoff et al. 2012). These values were chosen in accordance with previous analysis but could also have been discussed; however, emphasis was placed in this study on the role of discontinuity grouping methods.

In this particular case, the slope is bolted (Gasc-Barbier et al. 2008); the stability analysis without bolting and with unfavourable mechanical properties is only used as a reference. If we compared the models with the bolt pattern, most models would have likely lead to no unstable blocks and a zero volume of unstable blocks; thus, no comparison would have been available. We could have looked at the 


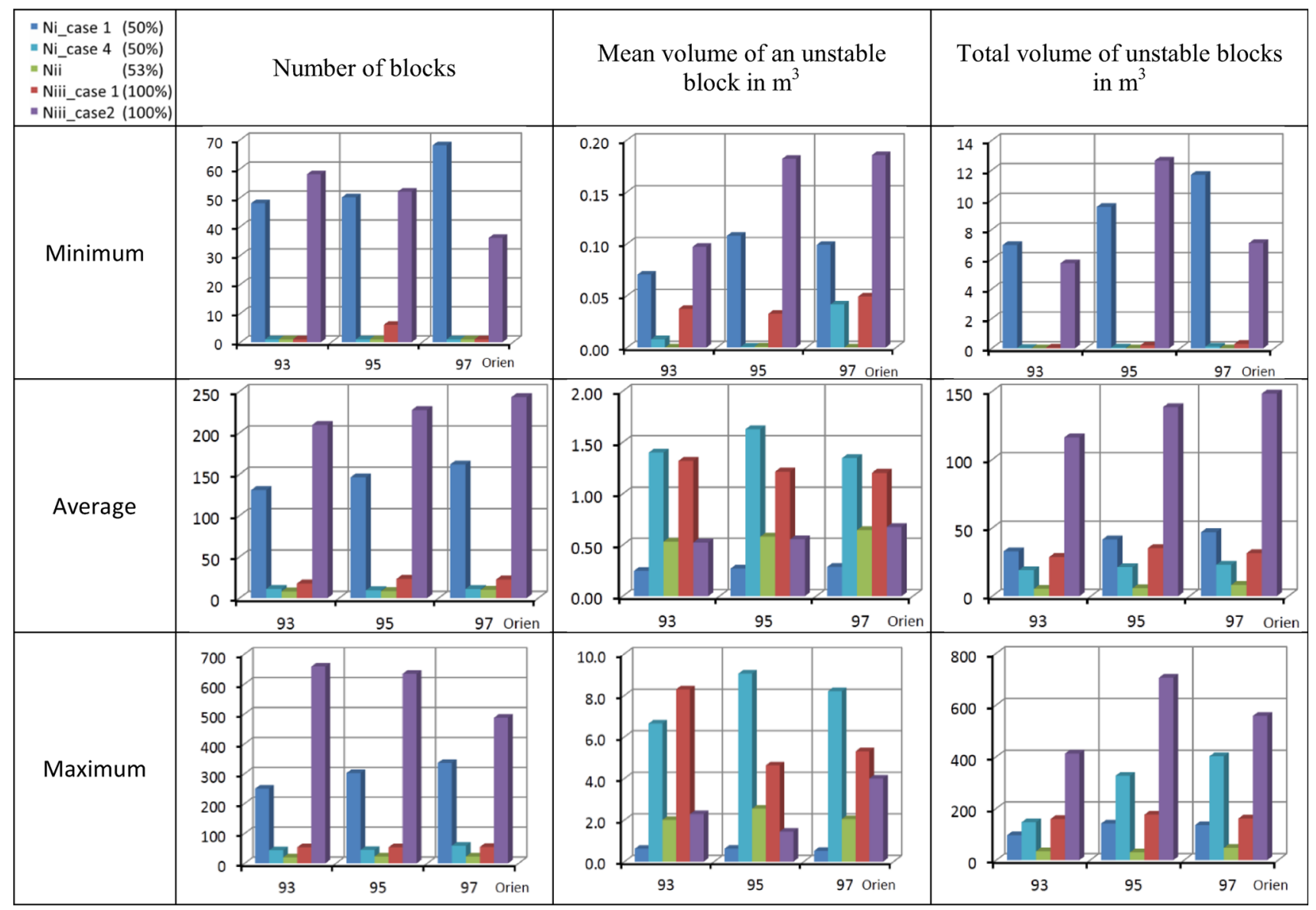

Fig. 10 From left to right, evaluation of: the number of unstable blocks, the mean volume of an unstable block, the total volume of unstable blocks. From top to bottom, the minimum, average, and maximum values of the 50 simulations performed in this study. The

effect of different bolting patterns, but this also would have taken us away from the focus of this study.

The RESOBLOK software calculates the safety factor $\mathrm{F}$ of the blocks around the excavation, and those with a safety factor lower than a fixed value (default value $=1$ ) are considered to be unstable.

Removing blocks with a safety factor $\mathrm{F}$ greater than 1 allows for a more "secure" analysis because it enhances the number and volume of unstable blocks; this process is equivalent to the reduction of $\mathrm{C}$ and $\varphi$ of the discontinuities by dividing them by $\mathrm{F}$. It is also possible to decrease the safety factor below 1 (and thus increase $\mathrm{C}$ and $\varphi$ ) until no blocks are unstable, or a few blocks are unstable; this option will be used below to allow the computation of a global unstability factor.

(a) Influence of the grouping method used on the stability analysis results

Stability analyses were performed with five of the seven grouping cases from Table 1 using the DIPS (Ni_case1 and five coloured boxes correspond to the five regrouping processes, and for each regrouping process, $93^{\circ}, 95^{\circ}$, and $97^{\circ}$ are the slope dip directions that correspond to the possible orientations of the cut (e.g., $3^{\circ}, 5^{\circ}$ and $7^{\circ}$ )

Ni_case4), PSMY (Nii), and spectral methods (Niii_case 1 and Niii_case 2). For each regrouping process, three orientations of the slope were tested (i.e., $3^{\circ}, 5^{\circ}$ and $7^{\circ}$ ). For each scenario (i.e., a regrouping method and a slope orientation), 50 simulations were run, and the minimum, mean and maximum number, mean volume and total volume of unstable blocks for the scenario were determined. The synthesis of the 750 simulations is shown in Fig. 10 in a bar graph where each colour represents a regrouping process, and the three possible orientations cases are presented in succession. An example of the output from a simulation using the spectral method grouping (Niii_cas1) and an orientation of the slope of $5^{\circ}$ is shown in Fig. 11 .

The bar heights (Fig. 10) vary significantly from one scenario to another, showing the influence of the fracture data processing and orientation of the slope on the stability indicators. The most (or least) favourable case also depends on the criterion used to evaluate the slope stability (e.g., the lowest average block volume or the smallest maximum block volume, etc.). The case Ni-Case4 (in green on 
Fig. 11 Example of unstable blocks for the level-III treatment of the data (Niii_cas1) on the $\left(5^{\circ} \mathrm{N} 75^{\circ}\right)$ cut with the most favourable simulation in RESOBLOK

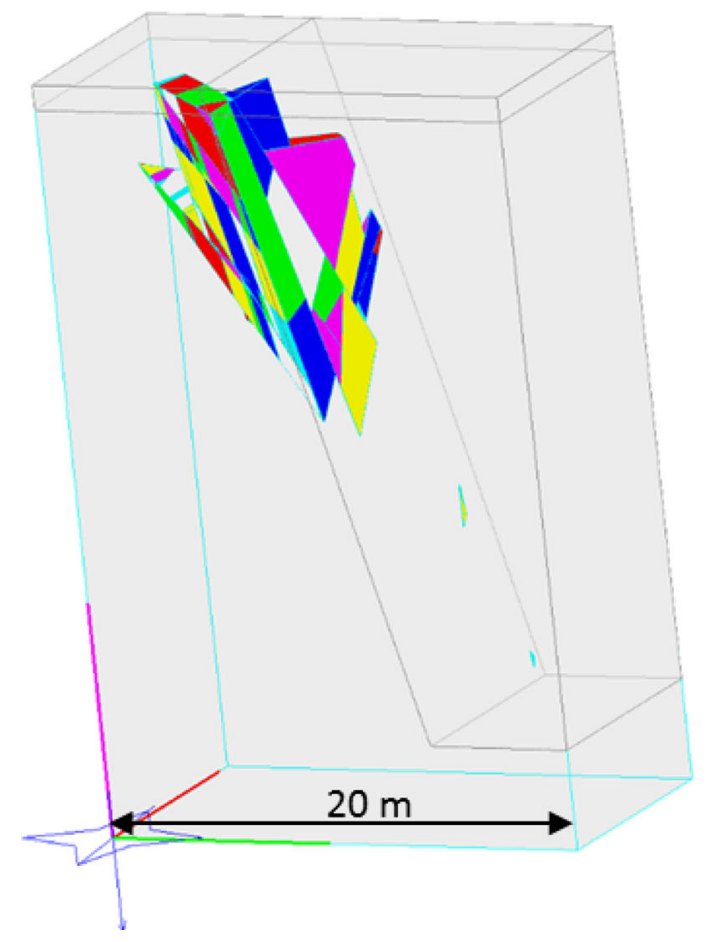

Fig. 10) could not be compared to the other cases, as described in "Structural study and statistical treatments" section.

For the spectral regrouping method, it is possible to compare the influence of regrouping into three (Nii-case1) or four (Niii-case2) families by comparing the last two cases of the bar graphs. Considering three families leads to a larger number of unstable blocks and a larger total volume of blocks; however, as discussed in "RESOBLOK software" section, a smaller mean volume of the unstable blocks is also shown. The most favourable case leads to a minimum of the mean volume of an unstable block, which is approximately $0.05 \mathrm{~m}^{3}$ and thus remains rather important.

For the Ax-les-Thermes case, regrouping into three families therefore leads to better stability indicators than regrouping into four families, which may be considered as obvious because considering more families tends to produce more cuts in the rock mass in various directions. However, aggregation into families also influences the spacing statistics; more families decrease the mean spacing of each family.

In this example, the grouping method used and the stability indicator considered can lead to significantly different conclusions. It is also difficult to determine the best orientation of the slope cuts based on the security, considering the proposed set of indicators. In fact, if we compare the middle line of Fig. 10, which describes the "average" values of the number of blocks and the total volume of an unstable block, $3^{\circ}$ seems the most favourable orientation of the slope, producing fewer blocks and a smaller volume; however, if we if we rely on the maximum values (i.e., the next line), the conclusion is not as clear. The goal of this study is not to provide decision criteria but to note that there are different stability indicators, corresponding to different hazards (i.e., more frequent or less probable hazards).

Thus, these bar graphs provide the orders of magnitude of the different stability indicators, which can be compared to other cases (e.g., the same case with bolts).

\section{(b) Analysis of variance}

The Analysis of Variance (ANOVA) is a statistical technique used to test differences between two or more means. In this study, to compare and highlight the effect of the two factors taken into consideration: the statistical-fracture processing (i.e., grouping level) and the bench orientation, the means of the different stability indicators were compared.

The five fracture groupings treated in the previous section and the three possible bench orientations of the cut $\left(3^{\circ}\right.$, $5^{\circ}$ and $7^{\circ}$ ) were considered. The ANOVA was computed using the three stability indicators (i.e., the number of unstable blocks, the mean volume of an unstable block, and the total volume of unstable blocks). First, a single-factor (i.e., one-way) ANOVA is used to verify that each factor (i.e., the grouping level and the bench orientation) influences the stability indicators; then, a two-way ANOVA is 
Table 2 Results of ANOVA: influence of the level of data treatment and three orientations of the cut on the number, the mean volume, the total volume of unstable blocks

\begin{tabular}{|c|c|c|c|c|c|c|c|}
\hline \multicolumn{2}{|c|}{ Values } & \multicolumn{4}{|l|}{$D F=2$} & \multirow{2}{*}{\multicolumn{2}{|c|}{$\begin{array}{l}D F=3 \\
\text { Orientation of slope and level of data treatment }\end{array}$}} \\
\hline & & \multicolumn{2}{|c|}{ Orientation of the slope $(O)$} & \multicolumn{2}{|c|}{ Level of data treatment $(N)$} & & \\
\hline & & $F$ value & $P$ value & $F$ value & $P$ value & $F$ value & $P$ value \\
\hline \multirow[t]{3}{*}{$N_{\mathrm{m}}$} & Minimum & 0.0348 & 0.9659 & 66.269 & 0.000080 & 3.259 & 0.38174 \\
\hline & Average & 0.0198 & 0.9804 & 220.593 & 0.000002 & $4.9 \times 10^{26}$ & 0. \\
\hline & Maximum & 0.0301 & 0.9705 & 107.371 & 0.000020 & 1355.070 & 0.01990 \\
\hline \multirow[t]{3}{*}{$V_{\mathrm{m}}$} & Minimum & 0.0712 & 0.9320 & 18.8731 & 0.002580 & 1.321 & 0.55168 \\
\hline & Average & 0.0269 & 0.9735 & 81.093 & 0.000045 & 139.956 & 0.06203 \\
\hline & Maximum & 0.1147 & 0.8934 & 10.505 & 0.010961 & 11.595 & 0.21184 \\
\hline \multirow[t]{3}{*}{$V$} & Minimum & 0.0687 & 0.9342 & 46.116 & 0.000228 & 414.066 & 0.03610 \\
\hline & Average & 0.1423 & 0.8701 & 37.700 & 0.000400 & 1.086 & 0.59180 \\
\hline & Maximum & 0.1979 & 0.8255 & 18.421 & 0.002746 & 3.176 & 0.38610 \\
\hline
\end{tabular}

One-way ANOVA with degree of freedom $D F=2$, and two-way ANOVA with $D F=3 . F$ test and associated probability. Italicized values are correspond to a $P$ value lower than $5 \%$, and bolded $P$ values are lower than $3 \%$

used to compare the effect of each factor and whether the factors interact. The ANOVA compared the variance of the groups to the entire variance using the Fisher ratio $(F)$. Generally, the influence of a factor is considered to be significant when the probability (i.e., $P$ value) of the observed Fisher ratio $(F)$ is lower than $0.05(5 \%)$.

The one-way ANOVA (Table 2) indicates that the factor "orientation of slope cuts" is not significant and that the factor "level of data treatment" does significantly affect the stability indicators except for the maximum mean volume of an unstable block.

The two-way ANOVA (Table 2) completes the analysis by showing the interactions between the two factors for nearly all of the stability indicators investigated in this study.

Globally, the ANOVA shows that the orientation of the slope cuts for the low variation used in this study has no significant influence on the stability indicators, while the level of the data treatment has a significant impact. The variation of orientation considered in this study was the possible variation of the orientation of the cut. In this example, the variation is very low, and this result should certainly not be extrapolated to cases where the variation of the orientation of the slope is greater.

The influence of interaction between slope orientation and fracture orientation is a well-known result, but the interaction between statistical-fracture processing and slope orientation is only marginally supported by the ANOVA performed in this study perhaps due to the low variation of the slope orientation.

(c) Global stability assessment

Although the numerous results given by RESOBLOK can provide various perspectives on slope stability, the slope stability safety factor $F$ was gradually decreased until no blocks were unstable to get a unique global indicator. This use of the safety factor is unusual because it lead to a safety factor lower than 1; however, this representation expresses the demand that is still required to make the slope stable. In Fig. 12, we use the inverse of the safety factor called the unstability factor (UF).

Considering the mechanical properties of the joints suggested at the beginning of this section, the decrease of the safety factor only influences the friction angle because $C=0$, and Fig. 12 shows the increase in the unstability factors when the friction angle increases. For a decreasing safety factor, the distribution of the number, mean volume, and total volume of unstable blocks in $\mathrm{m}^{3}$ (Fig. 12) versus the friction angle on a $5^{\circ}$ oriented bench are shown using the various grouping methods. The stability could be considered to be reached when all stability indicators equal zero. In this study, it corresponds to an improbable friction angle on site, which confirms the need of bolting.

This figure also highlights the strong variation between 1 and 10 of the mean volume of the unstable blocks based on the chosen grouping method with a safety factor $(F)$ of 1. This result suggests that a low or high estimation of the unstable blocks between 1 and 10 could be calculated based on the various grouping method used.

The UF value that leads to no unstable blocks varies from 5.1 to 10.0 based on the grouping method used; this value can be considered to be "the support need."

In situ, no unstability was observed because the slope was reinforced with bolts that provide traction resistance equal to $241 \mathrm{kN}$ (Gasc-Barbier et al. 2008) and a density of 1 bolt $/ 7.5 \mathrm{~m}^{2}$, which could be approximated as an additional global cohesion of $32 \mathrm{kPa}$. However, as explained by Gasc-Barbier et al. (2008), adding a global cohesion to every fracture is not appropriate because bolts may or may not cross different fractures in different ways. 

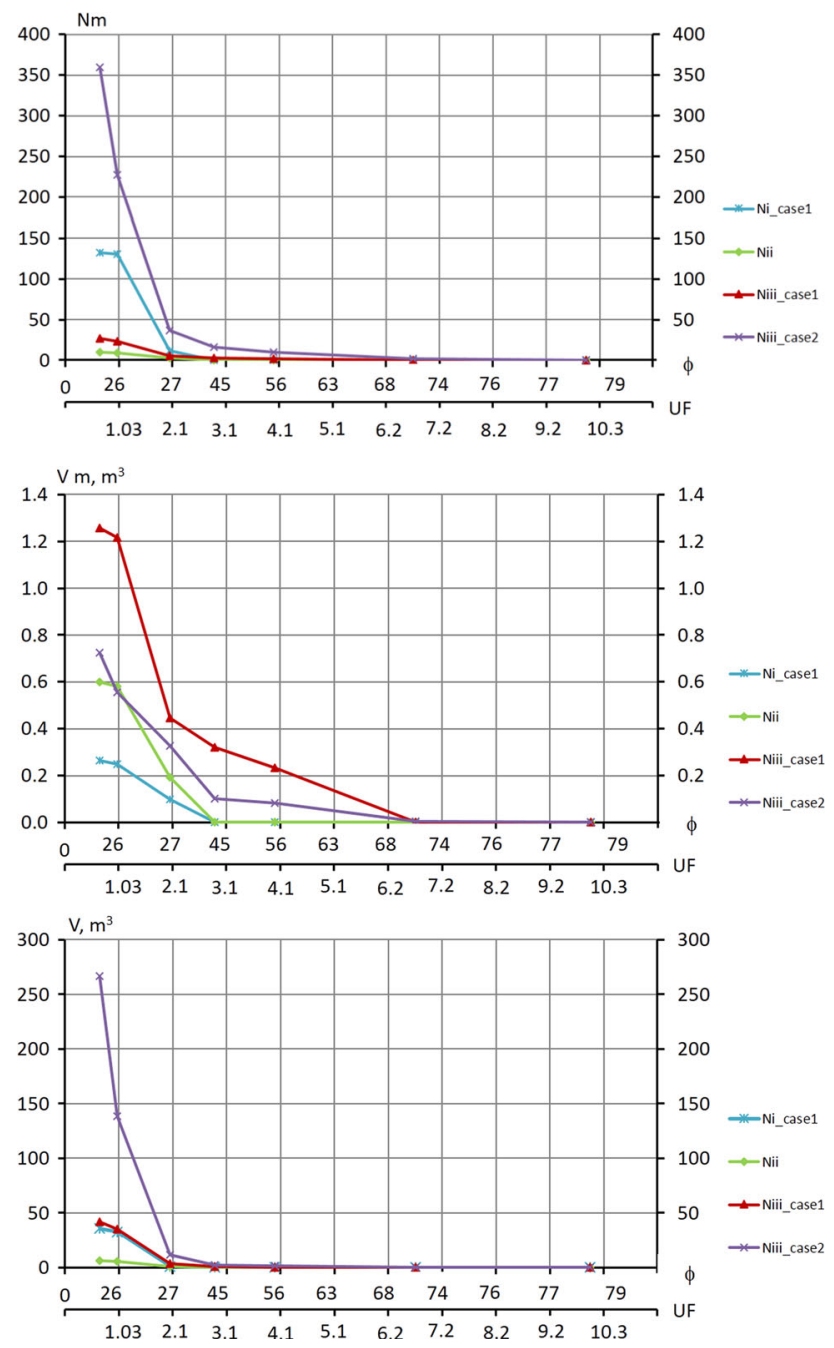

Fig. 12 Distribution of the mean number of unstable blocks $(\mathrm{Nm})$, the mean of the volume of unstable blocks $(\mathrm{Vm})$ and the mean unstable blocks volume (V) versus the friction angle of the discontinuities $(\varphi)$ or the unstability factor (UF) for the four cases of data (Ni_case1, Nii, Niii_case 1 and Nii_case 2) regrouping on the $5^{\circ}$ oriented cut

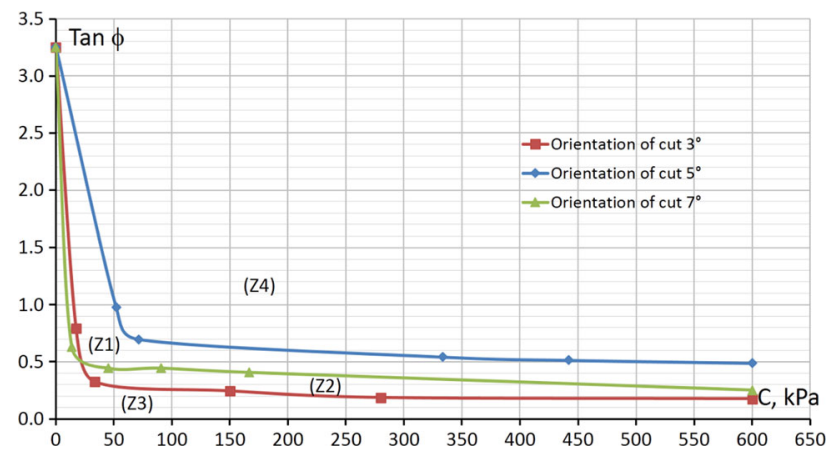

Fig. 13 Limit of the stability curves on the three orientations of the cut $\left(3^{\circ}, 5^{\circ}\right.$, and $\left.7^{\circ}\right)$ at level treatment Niii_cas1 in a $\tan (\phi)$ versus the cohesion of the discontinuities graph for the 50 simulations
Finally, varying the mechanical properties of the discontinuities (i.e., $C$ and $\varphi$ ), the cut azimuth and the grouping method used allow for different zones of stability to be defined: $Z 4$ represents a stable zone for any cut orientation; $Z 3$ represents an unstable zone for any cut orientation; and $Z 1$ and $Z 2$ represent stable zones for only certain cut orientations (Fig. 13).

\section{Conclusions}

Many factors influence the stability of a rock slope. The impact of various uncertainties on final stability analysis is seldom investigated. In this study, we investigated the role of grouping discontinuity methods on stability simulations and stability indicators, such as the number of unstable blocks, the mean volume of an unstable block, and the total volume of unstable blocks.

Three grouping methods of discontinuities into primary sets were presented. Each method showed different advantages. The PSMY method allows for the automatic determination of the optimal number of families to be adopted, but a proportion of discontinuities are not assigned to a families (i.e., $47 \%$ in the case investigated in this study). The spectral method attributed each discontinuity to a family, but the number of families must be initially given, possibly using from the PSMY method, and the grouping depends on a factor $\sigma$, whose sensitivity was examined in this study. The manual method using DIPS software is convenient and useful, and can consider qualitative and geological information; however, it is broadly dependent on the user's skills and experience, and can produce variable results. Certain discontinuities are also not classified into any family. As a result, the stochastic geometric simulations produced by any of the statistics not classifying all the discontinuities will be biased.

The three grouping methods were applied to a rock cut near Ax-les-Thermes, and the statistical parameters of the families were computed using 856 measured discontinuities from 11 scan lines.

The different combinations of statistical parameters were used to generate a realistic, 3D, fractured rock-mass model and a stochastic stability evaluation of the cut in the fractured rock site. It was shown that the results significantly depended on the grouping method used, and in this case, are influenced only slightly by a weak $\left(2^{\circ}\right)$ variation in the cut orientation. The evaluation of the mean total number of unstable blocks and the mean unstable block volume can vary from 1 to 100 based on the grouping method used.

Modelling also led to the definition of zones of global stability or unstability, depending on the cohesion, friction angle, and the grouping process used. 
Three different stability indicators were used to evaluate stability: the number of unstable blocks, the mean volume of an unstable block, and the total volume of blocks. It was shown that the safer or the less safe case also depends on the chosen indicator and that the choice between them is not obvious.

The stability indicators were compared to a non-bolted slope when, in reality, the slope is bolted; this situation was performed because it enhanced the differences, but the methodology could also be applied to compare different bolting patterns.

In the case study at Ax-les-Thermes, the influence of different automatic grouping processes on the stability analysis were shown to be of the same order of magnitude as the influence of considering geological considerations using a fourth family. More precise knowledge of the genesis of the fracture network could perhaps be the best way to reduce uncertainties; however, significant amounts of work remain to connect the structural analysis and its use by the slope designer.

The role of a hierarchical rule between fractures, which is an important factor, was also discussed but not fully investigated in this study. However, this role can be more important than the choice of the grouping method. In addition, the fractures in this study were assumed to completely cut the blocks; the influence of partial cut of blocks by fractures could not be investigated with the software used in this study.

To consider all of these influences simultaneously on a significant number of simulations will be required to perform a comprehensive ANOVA with several factors.

Acknowledgments The authors wish to thank M. Thierry Verdel, Professor GéoRessources Laboratory, Ecole des Mines de Nancy, Université de Lorraine, France, for providing help with the Mathematica software used in this study.

\section{References}

Andrew YN, Michael IJ, Weiss Y (2002) On spectral clustering: analysis and an algorithm. In: Advances in neural information processing systems, vol 14. MIT Press, Cambridge, pp 849-856

Baroudi H, Hantz D, Asof M, Piguet JP (1992) Bench stability in open pit mines: a methodology for jointed rock masses. In: Regional conference on fractured and jointed rock masses

Barton NR (2012) From empiricism, through theory, to problem solving in rock engineering. In: Qian Q, Zhou Y (eds) Harmonising rock engineering and the environment. Taylor \& Francis Group, London, p 13

Diederichs MS (1990) Dips an interactive and graphical approach to the analysis of orientation based data. Univercity of Toronto, Toronto

Gasc-Barbier M, Ballion A, Virely D (2008) Design of large cuttings in jointed rock. Bull Eng Geol Environ 67(2):227-235

Gasc-Barbier M, Fouché O, Gaillard C (2010) Etude comparée de la fracturation observable sur carottes de forage et obtenue par diagraphie. Application au marbre de Saint-Béat (HauteGaronne). Rev Fr Géotech 133:37-49

Godefroy J, Merrien-Soukatchoff V, Gasc-Barbier M (2009) Stabilité de pentes rocheuses fracturées. Recherche des conditions aux limites pour un calcul à l'équilibre limite 3D. Journées AUGC 2009, p 17

Hammah RE, Curran JH (1998) Fuzzy cluster algorithm for the automatic identification of joint sets. Int J Rock Mech Min Sci 35(7):889-905

Hammah RE, Curran JH (1999) On distance measures for the fuzzy K-means algorithm for joint data. Rock Mech Rock Eng 32(1):1-27

Heliot D (1988) Generating a blocky rock mass. Int J Rock Mech Min Sci Geomech Abstr 25(3):127-138

Jimenez-Rodriguez R (2008) Fuzzy spectral clustering for identification of rock discontinuity sets. Rock Mech Rock Eng 41(6):929-939

Jimenez-Rodriguez R, Sitar N (2006a) A spectral method for clustering of rock discontinuity sets. Int J Rock Mech Min Sci 43(7):1052-1061

Jimenez-Rodriguez R, Sitar N (2006b) Influence of stochastic discontinuity network parameters on the formation of removable blocks in rock slopes. Rock Mech Rock Eng 41(4):563-585

Kabbaj R, Baroudi H (1995) Traitement statistique de la fracturation. In: Séminaire Formation Modelisation des Milieux Discontinus. Ecole des Mines de Nancy, Nancy, France, p 42

Klose C, Seo S, Obermayer K (2005) A new clustering appproach for partitioning directional data. Int $J$ Rock Mech Min Sci 42(2):315-321

Mahtab MA, Yegulalp TM (1982) A rejection criterion for definition of clusters in orientation data. American Rock Mechanics Association, The 23rd U, p 8

Mardia KV (1975) Statistics of directional data. J R Stat Soc Ser B Methodol 37(3):349-393

Meila M, Shi J (2001) Learning segmentation by random walks. In: neural information proceedings systems, vol 13, p 7

Merrien-Soukatchoff V, Korini T, Thoraval A (2012) Use of an integrated discrete fracture network code for stochastic stability analyses of fractured rock masses. Rock Mech Rock Eng. doi:10. 1007/s00603-011-0136-7

Mirkin B (1996) Mathematical calssification and clustering. Kluwer Academic Publishers, Moscow

Pecher A (1989) SCHMIDTMAC — a program to display and analyze directional data. Comput Geosci 15(8):1315-1326

Picard P (1999) Classification sur des données hétérogènes. Université de la Réunion, Réunion

Priest S (1993) Discontinuity analysis for rock engineering. Chapman \& Hall, USA

Rafiee A, Vinches M (2008) Application of geostatistical characteristics of rock mass fracture systems in 3D model generation. Int $\mathrm{J}$ Rock Mech Min Sci 45(4):644-652

Rockscience Inc (2002) DIPS Plotting, Analysis and Presentation of Structural Data Usinng Spherical Procjection Techniques.(C) 1989-2002 Rocscience Inc.

Shanley RJ, Mahtab MA (1976) Delineation and analysis of clusters in orientation data. Math Geol 8(1):15

Verdel T (1999) Le logiciel STAF. Nancy, France

Warburton PM (1981) Vector stability analysis of an arbitrary polyhedral rock block with any number of free faces. Int J Rock Mech Min Sci Geomech Abstr 18(5):415-427

Wu J, Zhang ZX (2013) Cluster analysis for orientation data using difFUZZY method. In: Huang Y et al (eds), p 5

Wyllie DC, Mah CW, Hoek E (2004) Rock slope engineering. Taylor \& Francis Group, London 